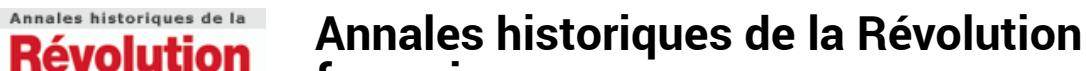

française française

352 | avril-juin 2008

Les temps composés de l'économie

\section{Des contrats sous tension : rétablir la propriété} après la Terreur

Contracts Under Pressure: Property Recovery after the Terror in France

Judith A. Miller

Traducteur : Sophie Bond et Philippe Minard

\section{OpenEdition}

Journals

Édition électronique

URL : https://journals.openedition.org/ahrf/11134

DOI : 10.4000/ahrf.11134

ISSN : 1952-403X

Éditeur :

Armand Colin, Société des études robespierristes

Édition imprimée

Date de publication : 1 juin 2008

Pagination : 241-262

ISBN : 978-2200-92514-7

ISSN : 0003-4436

Référence électronique

Judith A. Miller, «Des contrats sous tension : rétablir la propriété après la Terreur », Annales historiques de la Révolution française [En ligne], 352 | avril-juin 2008, mis en ligne le 01 juin 2011, consulté le 23 avril 2022. URL : http://journals.openedition.org/ahrf/11134; DOI : https://doi.org/10.4000/ahrf.11134 


\section{DES CONTRATS SOUS TENSION : RÉTABLIR LA PROPRIÉTÉ APRÈS LA TERREUR}

Judith A. MILLER

Cet article étudie l'hyperinflation (1791-1797), qui éviscéra le patrimoine de la plupart des familles, et un remède à celle-ci, aussi inattendu que controversé : I'« action en rescision pour lésion d'outremoitié ». Pendant que l'assignat tombait en-dessous d'1 \% de sa valeur nominale, des familles affolées vendirent boutiques, maisons ou domaines afin de survivre à la crise. Souvent, ces propriétés furent cédées bien en-dessous de leur valeur, conduisant les hommes de loi à ressusciter cette procédure ancrée dans la loi romaine. Elle accordait aux vendeurs le droit de demander à leurs acheteurs une réévaluation du montant de la vente. Des débats enflammés secouèrent la société française et remirent en question plusieurs milliers de ventes, notamment après la loi du 19 floréal an VI. Les 531 actions trouvées dans les archives des Yvelines témoignent de la férocité avec laquelle les vendeurs poursuivirent leurs acheteurs. Au-delà de ces poursuites judiciaires, c'est la notion même de contrat qui était en débat : sa légitimité procède-t-elle de l'accord des volontés ou d'une mesure de l'équité ?

Mots-clés : hyperinflation, propriété, patrimoine, droit civil, théorie du contrat, assignat, période thermidorienne.

« Or, de ce que tout individu est maître absolu de sa personne, il suit que la liberté pleine de son consentement est une condition inséparable de tout contrat et de tout engagement $»$.

(Volney, Les ruines, 1799, p. 120) 
Paris, février 1795, six mois après le renversement des Jacobins ${ }^{1}$. Les députés de la Convention qui siègent encore annulent les lois votées sous la Terreur, ferment les tribunaux révolutionnaires et libèrent leurs prisonniers, mais ils peinent à établir de nouvelles bases pour la République et à consolider les acquis de la Révolution.

Cet hiver-là est exceptionnellement rude ; à la grêle et aux mauvaises récoltes de 1794 succède un froid glacial. L'augmentation du prix du pain (lorsqu' on peut se procurer de la farine) surpasse largement celle des salaires ; en 1795, la miche coûte neuf fois plus cher qu'en 1790. En résumé, les souffrances endurées sont terribles. La police rapporte que les populations affamées attendent, pieds nus dans la neige, pour quelques onces de grain ou se jettent sur une carcasse de cheval en décomposition, si grand est leur désespoir. Ce climat d'angoisse ne laisse présager rien de bon quant à la suite des événements : guerre permanente en Europe, effondrement économique et crises constitutionnelles.

Des travaux récents ont largement contribué à améliorer notre compréhension de cette période de troubles ${ }^{2}$. Une attention plus particulière a été portée aux incessantes querelles qui divisaient le pays et les législatures entre droite et gauche, entre contre-révolutionnaires, royalistes, républicains, jacobins et catholiques. Des études menées à l'échelle régionale ont révélé l'intensité des explosions de violence dans de nombreuses régions du Sud et de l'Ouest ; une situation qui conduisit le gouvernement à adopter des mesures plus autoritaires. La réaction du gouvernement apparaît à la fois compréhensible et profondément dérangeante, car dans sa lutte pour le rétablissement de l'ordre, il a recours à des moyens de plus en plus illégitimes. Pour certains historiens, les coups d'État, les purges, l'annulation des élections et l'exil de législateurs - et non l'État de droit - caractérisent la période qui mène de la Terreur au

(1) Je tiens à remercier A. Rosenbaum, J. Dunn, K. Fidler, N. Starostina, et E. Schiff pour leur aide ; ainsi que les institutions suivantes : départment d'histoire de l'université d'Emory, University Research Committee, Institute for Comparative and International Studies, European Studies Seminar ansi que le Fox Center for Humanistic Inquiry, pour leur soutien financier. Le personnel des archives des Yvelines m'a fourni une aide très précieuse. Cet article développe une recherche déjà évoquée : «The aftermath of the assignat : plaintiffs in the age of property, 1794-1804, » dans Howard G. Brown, Judith A. Miller (eds.), Taking Liberties: The Problems of a New Order in France, 17941804, Manchester, Manchester University Press, 2003, p. 70-91.

(2) Parmi les ouvrages clefs : Michel Vovelle (éd.), Le tournant de l'an III: Réaction et Terreur blanche dans la France révolutionnaire, Paris, Paris, Éditions du CTHS, 1997 ; Jean-Clément Martin, Contre-révolution, révolution, et nation en France, 1789-1799, Paris, Points-Seuil, 1998 ; Jacques Bernet, Jean-Piere Jessenne, Hervé Leuwers et alii (éds.), Du Directoire au Consulat, Villeneuve d'Ascq, Centre de recherche sur l'histoire de l'Europe du Nord-Ouest, 1999-2001, 4 vol. 
Directoire et jusqu'à Bonaparte (1795-1799). Mais à côté des expulsions politiques et des élections contestées, une question tout aussi importante a cependant été insuffisamment explorée : la violente hyperinflation survenue au milieu des années 1790, et ses conséquences multiples.

\section{L'hyperinflation révolutionnaire, 1791-1797}

On dispose de quelques études majeures sur la création de devises en papier-monnaie, d'abord l'assignat puis le mandat territorial, et sur la chute vertigineuse de leur cours entre 1791 et la fin de l'année $1797^{3}$. Lorsqu'en décembre 1794, la Convention post-Terreur décida d'abolir la loi sur le Maximum, signifiant une possible fin de l'économie dirigée, la valeur de ces devises dégringola brusquement, et à l'automne 1795, celle de l'assignat discrédité ne montait plus qu'à $1 \%$ de sa valeur nominale.

Dans les bourgs modestes, les familles, qui peinaient pour payer leur miche de pain noir, se demandaient si le boulanger consentirait à leur accorder une semaine de crédit supplémentaire. Contemplant leurs maigres possessions - un plateau d'argent terni, une montre, ou un miroir qu'ils conservaient précieusement - elles se demandaient quelle somme elles pourraient en retirer si toutefois elles parvenaient à trouver un acquéreur. Les foyers bourgeois réalisèrent qu'ils n'étaient pas plus à l'abri de l'inflation que ne l'étaient les plus déshérités : leurs solides calculs de 1770 d'un rapport régulier de $5 \%$ sur leurs rentes s'étaient révélés douloureusement inexacts. Leurs débiteurs avaient surgi, jubilant, pour leur donner du papier-monnaie sans valeur, et se déclarer ainsi acquittés des dettes qu'ils avaient contractées en argent ou en or. Ces malheureux créanciers ne pouvaient rien faire pour protester contre l'injustice dont ils pensaient avoir été victimes. En avril 1793, la Convention avait déclaré illégale l'utilisation du louis et de l'écu, qui rappelaient trop l'Ancien Régime. D'après l'un des députés de l'an V, la Terreur avait arraché les devises en métal des mains des Français pour les remplacer par du vulgaire papier leur offrant comme unique et rude choix : « les assignats ou la mort $»^{4}$.

(3) François CRouzet, La grande inflation: La monnaie en France de Louis XVI à Napoléon, Paris, Fayard, 1993. Voir aussi Philip T. Hoffman, Gilles Postel-VinaY, Jean-Laurent Rosenthal, Des marchés sans prix : une économie politique du crédit à Paris, 1660-1870, Paris, Éditions de l'EHESS, 2001.

(4) Archives nationales (désormais AN), C 496, fol. 110, n 330, 18 nivôse an V, C.-F. Lebrun, Rapport au Conseil des Cinq-Cents. 
Le vent tourna après le 16 pluviôse an $\mathrm{V}$, lorsque le gouvernement décida de supprimer l'utilisation du papier-monnaie pour revenir à celle de devises en métal. Une violente déflation suivit. Soudainement, les débiteurs qui avaient emprunté des sommes extravagantes en papier-monnaie dévalué, virent leurs créanciers résolus à leur soutirer la totalité de la somme en numéraire. Un prêt de dix mille livres en papier-monnaie, insistèrent-ils, devait être remboursé avec dix mille livres en pièces d'or ou d'argent. Les discussions que tenait alors le corps législatif sur la possibilité de rétablir l'emprisonnement pour dette ne firent qu'accroître le caractère terrifiant de la menace qui pesait sur les débiteurs ${ }^{5}$. D'un bout à l'autre de la République, des voix s'élevèrent pour appeler le gouvernement à agir.

Parmi toutes les transactions qui structuraient la société française - contrats de mariage, stipulations, successions, renonciations, obligations, rentes (viagères, perpétuelles, foncières) - la vente des biens désignés comme immobiliers était plus problématique que les autres, en cette période d'hyperinflation. Cette catégorie comprenait les bâtiments et terrains, et avait été élargie virtuellement afin d'englober les offices vénaux, et même les rentes, liées à leur acquisition. De façon symptomatique, le droit français, aussi varié fut-il, avec plus de soixante coutumes régionales et un droit écrit aux formes variables dans le sud du pays, ne concevait pas la propriété dépourvue de ses nombreuses caractéristiques. Les coutumes elles-mêmes étaient structurées selon ces catégories, traitant séparément la propriété ecclésiastique, seigneuriale ou maritale, ainsi que les héritages, notamment. Les juristes, conformément à l'usage consacré, firent preuve d'un soin minutieux dans l'examen des règles déterminant le transfert, le cumul ou l'achat de propriété dans chacune des régions. La propriété familiale elle-même ne faisait pas l'objet d'une catégorie distincte, puisqu'elle comprenait les biens provenant des lignées maternelles ainsi que paternelles, ou ceux qui avaient été ajoutés avant ou après le mariage dans le cadre du contrat ou à travers un héritage. Ce type de propriété ne pouvait être ni donnée, ni léguée, sans qu'une attention rigoureuse ne soit portée aux droits des enfants ou sans que soient prises en considération les revendications des générations plus âgées et celles des parents collatéraux.

(5) Voir les débats autour de la résolution du 12 ventôse an V concernant la contrainte par corps. 
Les lois révolutionnaires avaient dépouillé la propriété de nombre de ces attributs. Dans certaines circonstances, les frères, tout comme les sœurs, avaient des droits égaux sur la propriété de leurs parents ${ }^{6}$. Celle-ci avait perdu son statut d'acquêts ou de successorale cette dernière catégorie ne jouissant plus de la protection considérable que lui procuraient les lois d'Ancien Régime. À travers la France, les familles réunies dans leur salon firent le bilan des changements entraînés par la Révolution. Les femmes non mariées, les mères, les filles, les épouses, se réjouirent secrètement (ou ouvertement) de leur toute nouvelle possibilité d'accéder à la propriété en leur propre nom, que ce soit à travers un divorce, un héritage, ou via un achat effectué en vertu de leur nouveau pouvoir. Quant aux frères aînés, qui avaient escompté hériter des demeures ou des étables ancestrales, ils prirent sombrement conscience, en lisant les derniers débats parus dans le Moniteur universel du fait que, non seulement leurs jeunes frères, mais aussi leurs sœurs, avaient à présent un droit sur la propriété de leurs parents. Plus perturbant encore : ces lois étaient imposées au moment même ou l'hyperinflation s'accentuait. L'impact de l'hyperinflation sur la propriété familiale, et plus particulièrement sur les terrains et les demeures, mérite donc plus d'attention de la part des historiens.

Alors que la valeur de l'assignat chutait, des traités critiquaient le tort causé par l'inflation à toutes sortes de créanciers. Durant l'été 1795 , les deux assemblées résonnèrent de cris dénonçant l'indélicatesse de ces débiteurs qui pouvaient se libérer de dettes et de rentes grâce à un papiermonnaie dont la valeur chutait rapidement, alors que celles-ci auraient dû courir encore de nombreuses années. Horrifiés par la vitesse à laquelle ces dettes furent annulées cet été-là, les députés décidèrent, avec la loi du 25 messidor an III, de geler la totalité des remboursements, une mesure qui eut pour effet de supprimer tout revenu, quel qu'il soit, et de ruiner ainsi davantage les créanciers ${ }^{7}$.

(6) Voir plus particulièrement : La Révolution et l'ordre juridique privé : rationalité ou scandale? Actes du colloque d'Orléans 11-13 septembre 1986, Paris, PUF, 1988 ; Philippe BoucheR (dir.), La Révolution de la Justice : Des lois du roi au droit moderne, Paris, Jean-Pierre de Monza, 1989 ; Margaret H. Darrow, Revolution in the House: Family, Class, and Inheritance in Southern France, 1775-1825, Princeton, Princeton University Press, 1989 ; Jean-Louis HaLPÉRIN, L'Impossible Code civil, Paris, PUF, 1992; Xavier Martin, "Fonction paternelle et Code Napoléon », Annales historiques de la Révolution française, ${ }^{\circ} 305$, juillet-septembre 1996, p. 465-475; Susan DESAN, The Family on Trial in Revolutionary France, Berkeley, University of California Press, 2004; et Jennifer Ngaire Heuer, The Family and the Nation: Gender and Citizenship in Revolutionary France, 1789 1830, Ithaca, Cornell University Press, 2005.

(7) Moniteur universel (devenu le Moniteur), 30 messidor III. 


\section{La politisation de la perte}

«Souffrira-t-on que celui qui a reçu 100 1., par exemple, il y a six ans, en or, s'acquitte avec un petit écu ? " : c'est là bien sûr que le bât blesse $^{8}$. Que faire de toutes ces dettes qui avaient été remboursées à leur pleine valeur nominale mais en papier-monnaie ? La Convention pouvaitelle rester sans rien faire, alors que des dizaines de milliers de maisons, de boutiques et de fermes vendues pour des louis d'or avaient été remboursées avec des piles de papier sans aucune valeur? Tandis que les députés hésitaient à intervenir, les vendeurs se mirent quant à eux à chercher consolation dans les volumes moisis qui garnissaient les étagères des études d'avocats. En 1795, alors que la crise s'accentuait, remettant à l'ordre du jour une forme particulière d'action civile, d'audacieux jurisconsultes poussèrent ces vendeurs pleins de ressentiment à aller devant les tribunaux. Même s'ils pouvaient à peine la prononcer, et encore moins comprendre ses racines latines, une simple phrase avait fait renaitre tous les espoirs des plaignants qui, impatients, se mirent à réclamer une « action en rescision pour lésion d'outre moitié ».

L'idée sous-jacente était que le vendeur avait accepté un prix représentant moins de la moitié de la valeur présumée de la propriété au moment de sa vente ${ }^{9}$. Grâce à cette procédure, le vendeur espérait obtenir le juste prix avec intérêts. S'il gagnait l'affaire, le contrat était annulé, et l'infortuné acheteur n'avait plus que deux choix : payer, ou partir.

Alors que le nom de cette procédure semblait désespérément alambiqué, elle avait cependant des antécédents historiques incontestables. Tirant son origine du droit romain et solidement ancré dans le Corpus iuris civilis de Justinien, le concept de laesio enormis, au XVI ${ }^{\mathrm{e}}$ siècle, était accepté dans nombre de régions françaises. Il visait au départ, tout comme dans le droit romain, à protéger les mineurs qui avaient involontairement vendu à un prix excessivement bas les biens patrimoniaux ${ }^{10}$. De nombreux commentaires datant de l'Ancien Régime nous fournissent des informations sur cette procédure, la limitant à des cas d'héritage ou de requêtes émanant de mineurs, ou expliquant les points techniques qui déterminaient si seuls les vendeurs, et non les acheteurs, pouvaient y avoir

(8) Moniteur, 14 vendémiaire an IV, J.-F. Ehrmann, Convention.

(9) François Boulanger, « Le problème de la lésion dans le droit intermédiaire », Études d'histoire économique et sociale du XVIII siècle, 1966, n 9, p. 53-94.

(10) Jurisprudence de MM. Dalloz (1903), 3: 272-85. Hubert DE MESMAY, La nature juridique de la lésion en droit civil français, Thèse, Université de Paris II, 1980, p. 12-13. 
recours ${ }^{11}$. On vit réapparaître de telles actions de façon sporadique, et jusqu'en 1781, lorsque le parlement de Flandres traita une affaire venant du Hainaut ${ }^{12}$. Plus convaincante fut la contribution de Robert Joseph Pothier (1699-1772), le célèbre juriste d'Orléans, dont les volumineux traités faisaient l'un des principaux experts européens en la matière. S'appuyant sur le concept d'équité, il expliqua : « Un prix qui n'a aucune proportion avec la valeur de la chose vendue n'est pas un véritable prix $\rangle^{13}$. Bien que rarement utilisés, les cas de lésion, fondés à la fois sur le droit romain et sur le concept moderne d'équité, avaient une place évidente dans son univers jurisprudentiel.

Ces procès avaient été dénoncés par les physiocrates avant la Révolution car, selon eux, ils violaient le caractère sacré du consentement en matière de contrat, et il est évident que beaucoup s'attendaient à voir ces procédures disparaître avec l'Ancien Régime et son droit civil pesant et archaïque. Alors que les avocats et leurs clients commençaient à se pencher sur les éditions reliées de Pothier en quête d'une solution, un projet de code civil datant d'août 1793 proposait quant à lui d'abolir la lésion et l'équité comme motifs de contestation des ventes de propriétés. Un des premiers auteurs d'une des nombreuses tentatives d'élaboration d'un code civil, Jean-Jacques-Régis Cambacérès, expliqua que les contrats, y compris lorsqu'ils avaient été signés dans l'erreur, devaient demeurer valables ${ }^{14}$.

Alors que la crise s'accentuait à la fin de l'été 1795 , les députés rapportèrent que dans leurs provinces, des centaines de cas avaient été

(11) Jean-Baptiste DE Buridan, Coustumes de la cité et ville de Rheims, villes et villages régis selon icelles, Paris, chez Louis Billaine, 1665, p. 185-186; Arnoul FERRON, Conférence de toutes les questions traitées par Mr de Ferron... dans son commentaire sur la Coutume de Bordeaux... avec le commentaire de M. Bernard Automne,... contenant en outre plusieurs observations nouvelles... par feu Me Pierre Dupin, Bordeaux, J.-B. Lacornée, 1746, p. 188 ; Joseph-Nicolas GuYot, Répertoire universel et raisonné de jurisprudence civile, criminelle, canonique et bénéfciale, ouvrage de plusieurs jurisconsultes, publié... par M. G****..., Paris, Visse, 1784-1785, t. 10, p. 463-467; Gérard Trudel, Lésion et contrat, Montréal, Presses de 1'Université de Montréal, 1965; E. J. H. Schrage, Unjust Enrichment: The Comparative Legal History of the Law of Restitution, Berlin, Duncker \& Humblot, 1995; Reinhardt Zimmermann, The Law of Obligations: Roman Foundations of the Civilian Tradition, Oxford, Clarendon Press, 1996; et James Gordley, Foundations of Private Property Law: Property, Tort, Contract, Unjust Enrichment, Oxford, Oxford University Press, 2006.

(12) Joseph-Nicolas GuYot, Répertoire universel, op. cit., t. 10, p. 463-464.

(13) Robert Joseph Pothier, Traité du Contrat de Vente, dans Traités sur différentes matières

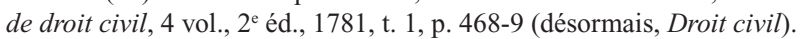

(14) François Boulanger, art. cit., p. 60-3. 
portés devant les tribunaux ${ }^{15}$. Finalement, le 14 fructidor an III, anxieux et frustrés, les membres de la Convention suspendirent toutes les poursuites en cours et interdirent le recours à ce type de procédure ${ }^{16}$. Cette décision jeta les plaignants potentiels dans un profond désespoir - propriété disparue, assignats sans valeur, remboursements des dettes gelés quelles que soient les circonstances, et voilà à présent que leur seul remède était remis en question.

Il serait trop long (et lassant pour le lecteur), de rapporter en détail les batailles législatives relatives aux accords commerciaux et à la propriété durant les deux ans et demi qui suivirent cette décision ${ }^{17}$. Les députés revinrent sans cesse à la question des transactions entre particuliers - une question « aussi ingrate qu'épineuse $»^{18}$ - et ce, bien après que le pays soit revenu au numéraire en 1797. Les délibérations autour des multiples formes d'arrangements possibles prirent des mois, pour mener le plus souvent à des impasses ou à des récriminations pleines d'emphase. Les enjeux politiques étaient très importants, car si un député soutenait que la valeur de l'assignat avait rapidement chuté, il affichait alors son manque de foi en la République. S'il faisait preuve de compassion envers les débiteurs, plaidant par exemple la cause de ceux qui risquaient l'emprisonnement, il s'attirait alors le courroux des créanciers. Si en revanche, il semblait prendre le parti des créanciers, les critiques venaient cette fois du corps législatif et de la presse, car cela signifiait qu'il reconnaissait la légitimité des plaintes supposées outrancières des vendeurs, ceux-là mêmes qui avaient immédiatement utilisé du papier-monnaie pour rembourser leurs dettes, et avaient ensuite l'audace de se plaindre d'avoir été trompés.

(15) Michel Marion, Histoire financière de la France depuis 1715, Paris, A. Rousseau, 1914-1931, vol. 3, p. 349-50 ; Boulanger, 66-7; Aristide DouARCHE, Les tribunaux civils de Paris pendant la Révolution (1791-1800), Paris, L. Cerf, 1905-07, t. 2, p. 53-4, 192-3, 153-4.

(16) Bulletin des Lois, 175, $\mathrm{n}^{\circ} 1061,31$ aout 1795.

(17) AN, C 489-496. Le 5 frimaire an IV, les Conseils autorisèrent les parties à utiliser des devises en métal même si les créanciers ne pouvaient pas refuser le papier-monnaie. La loi du 12 frimaire an IV permit aux créanciers ayant des contrats datés d'avant le $1^{\text {er }}$ vendémiaire an IV de rejeter tout remboursement jusqu'à ce qu'une législation appropriée soit votée. Une fois les mandats territoriaux créés, la loi du 15 germinal an IV autorisa la reprise des remboursements. Après la chute du mandat, la loi du 29 messidor an IV suspendit à nouveau tous les paiements. Les Conseils autorisèrent les différentes parties à passer des accords aux conditions et avec les devises de leur choix (le 5 thermidor IV). La loi du 11 frimaire VI permit la reprise du remboursement des dettes. Judith A. Miller, «The aftermath of the assignat », dans Howard G. Brown, Judith A. Miller (eds.), Taking Liberties, op. cit.

(18) François-Michel VermeIL, Jean-François Fournel, Code des transactions ou Recueil complet des lois relatives aux obligations entre particuliers dans leur rapport avec le papier-monnaie..., Paris, Rondonneau, s. d., p. 36. 
Dans les deux assemblées, on débattait de la création d'un tableau qui indiquerait les taux mensuels, voire hebdomadaires, du papier-monnaie afin que les deux parties puissent recalculer les dettes ainsi que le montant des remboursements. De tels tableaux leur permettraient de recalculer les montants en assignats dépréciés, figurants dans leurs accords, et de les ramener à des valeurs raisonnables et, selon eux, équivalente en espèces.

Finalement, le 5 messidor an $\mathrm{V}$, il fut décidé d'établir dans chaque département un tableau de dépréciation, s'appuyant sur les prix et expertises locales ${ }^{19}$. Créanciers et débiteurs pouvaient ainsi se pencher sur les colonnes de ces tableaux, contrats datés en main et, vraisemblablement, se mettre d'accord sur les montants dus en numéraire. Les nombreuses formes de transactions - fermages, rentes de toutes sortes, obligations, hypothèques - nécessitaient cependant chacune un traitement différent. On vit se multiplier pétitions, pamphlets et protestations du public, réclamant des solutions ${ }^{20}$.

Cependant, à l'été de l'an $\mathrm{V}$, les débats sur le sort des accords conclus en assignats avaient été intégrés aux batailles de plus en plus intenses générées par l'élection, au printemps, d'un corps législatif plus conservateur. La chute du mandat territorial et le retour à l'usage de devises en métal encouragea les créanciers, qui désormais espéraient soutirer des paiements en or et en argent pour la pleine valeur nominale des contrats. Les députés conservateurs, méprisant la Révolution et son argent étaient de leur côté. Le texte adopté par le Conseil des CinqCents les 18 et 19 floréal an V offrait aux créanciers des possibilités encourageantes. Le vendeur d'immeubles pouvait insister pour que le contrat soit honoré de la façon suivante : un contrat de 5000 livres en assignats dépréciés devrait être payé par 5000 livres en espèces ${ }^{21}$. Cette loi, rejetée par les Anciens, tournait à l'avantage des seuls vendeurs ${ }^{22}$. Les députés conservateurs firent une dernière tentative, réussie, les 14 et 15 fructidor an $\mathrm{V}:$ le texte promettait aux créanciers que plusieurs types de dettes seraient entièrement remboursées en espèces ${ }^{23}$. À la veille du

(19) Bulletin des lois, 129, $\mathrm{n}^{\circ} 1254$; Pierre CARON, Tableaux de dépréciation du papier monnaie réédités, Paris, Imprimerie nationale, E. Leroux, 1909.

(20) Par exemple : Observations d'un rentier viager, qui a déjà été ruiné une fois..., Paris, Imprimerie de Teulières, s. d.

(21) AN, C 409, fol. 68, n 460, Conseil des 500, 18-19 floréal an V.

(22) Conseil des Anciens, 29 messidor V, Emmanuel CRETET, Rapport au nom de la commission spéciale...; Moniteur, 15 thermidor $\mathrm{V}, \mathrm{n}^{\circ} 315$.

(23) Moniteur, 22-23 thermidor an V, $\mathrm{n}^{\circ} 322-3$; et 20-21 fructidor an V, $\mathrm{n}^{\circ} 350-1$. 
18 fructidor an $\mathrm{V}$, les créanciers ont dû alors dormir tranquillement, rassurés par la ténacité de leurs partisans, rêvant de leurs biens et fortune reconquis.

Après le coup d'État des 18 et 19 fructidor an V, le second Directoire est parvenu à définir une série de solutions un peu plus modérées à l'interminable problème des transactions entre particuliers datant de la période du papier-monnaie. Les lois du 11 frimaire et 16 nivôse an VI établirent les procédures élémentaires quant aux diverses catégories de paiements et de réévaluations, fournissant également des consignes pour les ventes de biens ${ }^{24}$. Cette législation débloqua le remboursement de toutes les dettes encore gelées. Ainsi le corps législatif avait-il permis, avec la loi du 19 floréal an VI, la réouverture de milliers (voire des dizaines de milliers) de procès pour lésion, et autres plaintes, suspendus au cours de l'été $1795^{25}$. Les plaignants affluèrent devant les tribunaux, tandis que les juristes se bousculaient, quant à eux, pour proposer leurs services ${ }^{26}$. Des conseils devenus éminemment nécessaires, car ces lois demeuraient en grande partie profondément obscures. De telles poursuites pouvaientelles par exemple concerner les biens vendus par décret, aux enchères ou en tant que biens nationaux ${ }^{27}$ ? Les discussions durèrent une année, et l'on décida finalement qu'aucune de ces catégories n'entrait dans le cadre de ces poursuites ${ }^{28}$. Les vendeurs se ruèrent devant les tribunaux, s'entêtant à énumérer les dommages que leur avaient causés les acquéreurs tandis que les greffiers, les doigts tachés d'encre, peinaient à faire face aux requêtes en citation des plaignants. Souvent, les acquéreurs étaient absents le jour de l'audience, même s'ils finissaient la plupart du temps par se montrer, indignés et incrédules, après avoir réalisé qu'ils prenaient le risque de perdre par défaut.

(24) Bulletin des lois, 161, $\mathrm{n}^{\circ} 1580$. Les clarifications au sujet des ventes de propriétés apparurent dans les lois du 16 nivôse an VI. Bulletin des lois, 174, $\mathrm{n}^{\circ} 1651$ et 1650 .

(25) La loi du 3 germinal an $\mathrm{V}$ avait permis la reprise temporaire des procès pour lésion. Analyse raisonnée de la loi du 19 floréal an 6..., Par F***, ancien jurisconsulte, dans François-Michel Vermeil, Jean-François Fournel, Code des transactions..., op. cit.

(26) Ibidem; QuesnEL, homme de loi, Sur le mode et les effets de la rescision..., Paris, Impr. de Renaudière, s. d. ; Pierre Bernaudaux, Décisions sur les ventes où il y a lésion..., Bordeaux, Imprimerie de Moreau, fructidor an VI.

(27) Meslier, La cause de tous les acquéreurs d'immeubles..., Paris, Petit, messidor an VI. Les plaignants tentèrent, sans succès, d'élargir la loi afin d'inclure les rentes perpétuelles. Gazette des Tribunaux, an V, t. XV, n 7, p. 247-249.

(28) Cette décision était en accord avec les conclusions de Robert Joseph PothiER, Droit civil, t. 1, p. 596-7; J. GrenIER, Conseil des Cinq-Cents, Second rapport fait [...] au nom de plusieurs commissions réunies, 7 pluviôse an VII; C.-J.-N. Duchatel dE LA Gironde, Conseil des 500, Opinion, 17 germinal an VII. 


\section{Litiges pour lésion en Seine-et-Oise}

«L'homme oublie plutôt le massacre de ses parents que le pillage de ses biens » (Cicéron $)^{29}$.

Aussitôt les lois promulguées en Seine-et-Oise, les vendeurs mécontents assiégèrent les tribunaux du département ${ }^{30}$. Cette région avait été durement affectée par la réorganisation judiciaire. Perruquiers, boulangers, marchands de vin et tailleurs avaient vu leur commerce s'effondrer dans les premières années de la Révolution. Le marasme s'étendit aux zones rurales, affectant également les propriétaires terriens aisés et les journaliers à peine solvables. On trouve au moins 531 actions intentées pour lésion dans les archives de Seine-et-Oise. Quelque 336 sont mentionnées dans les fonds des bureaux de paix, tandis que 228 sont citées dans les papiers du tribunal civil du département. En fait, les affaires de lésion représentent environ un tiers de toutes les affaires traitées par le bureau de paix de Meulan en l'an $\mathrm{VI}^{31}$. Treize références supplémentaires apparaissent parmi les registres notariaux, certaines indiquant l'enregistrement de nouveaux accords, d'autres confirmant les noms des fondés de pouvoir des plaignants dans l'attente d'une procédure.

La plupart du temps l'affaire débutait dans le bureau de paix ; le vendeur sommait l'acheteur de s'y présenter en vue de parvenir à un nouvel accord. À travers l'intervention des juges de paix, au moins vingttrois des plaignants de Seine-et-Oise réussirent à soutirer davantage d'argent à leurs acquéreurs, voire à récupérer leur propriété dans sa totalité. Le plus souvent cependant, l'acheteur insistait sur le fait qu'il avait payé la somme entièrement et dans les temps, refusant catégoriquement de payer davantage. Dans au moins 70 de ces cas, les acquéreurs prétendaient avoir payé plus que la somme mentionnée dans le contrat, affirmant - même si cela était illégal - avoir payé avec de l'or ou de l'argent, ou avoir également donné des sacs de café, de blé ou des barriques de vin. L'expression tranchante « non conciliation » ne peut entièrement rendre compte de la rancœur qui s'exprimait dans les bureaux de paix. Un greffier nota le mécontentement des juges face à un plaignant agité : « Bain s'est comporté indécemment en manquant de respect au Bureau par les diffé-

(29) Ciceron, De legibus, cité dans Encore un mot sur l'action en restitution pour cause de lésion d'outre moitié, s.l., s.d.

(30) Les sources utilisées ci-après proviennent des archives départementales (AD) des Yvelines (département créé en 1964).

(31) AD Yvelines, 64 L 42, Bureau de Conciliation, Meulan. 
rents emportements et gesticulations auxquels il s'est livré, le Bureau a invité ledit Bain à se retirer à peine d'y être contraint $»^{32}$. Alors que les gesticulations et les menaces d'emprisonnement ont été rares, les affirmations acrimonieuses ne l'étaient pas...

Quand les efforts du bureau de conciliation n'avaient pas permis de résoudre l'affaire, elle était transmise au tribunal civil du département. Dans ce cas, les juges ordonnaient systématiquement une estimation de la propriété (dans au moins 220 de ces cas, une expertise a été initiée). Chaque partie nommait ses experts, à qui incombait la tâche d'estimer la valeur réelle de la propriété. Souvent, l'expertise pouvait prendre plusieurs semaines, et malgré cela ne pas déboucher sur une nette victoire d'une des deux parties. En réalité, les experts se trouvaient en face d'une mission quasiment impossible. Plutôt que d'évaluer la valeur du bien par rapport à la valeur numéraire, plus stable et plus familière, tel que cela pouvait figurer auparavant dans n'importe quel contrat, ils devaient donner la valeur de l'immeuble en assignats, au moment de la vente et dans cette région, en se basant sur des transactions similaires. Cette mesure, qui constituait un obstacle non négligeable pour les plaignants, était un des éléments les plus modérés de la législation en vigueur depuis le 18 Fructidor. Les critiques se sont saisis de ce point, estimant que le fait d'ordonner une estimation était « diamétralement contraire au droit et à l'équité $»^{33}$. Après tout, très peu de plaignants auraient été en mesure de dénicher une autre propriété vendue dans la région et à un prix convenablement élevé en assignats, et ils étaient donc voués à perdre l'affaire ${ }^{34}$. Par conséquent, beaucoup dépendait du rapport de l'expert. Si les experts étaient consciencieux - et leurs notes de frais démesurées indiquent que beaucoup d'entre eux passèrent des mois à effectuer des estimations exhaustives - ils devaient non seulement traverser des champs plein de boue, mais également éplucher les registres de transactions conservés dans les études notariales à la recherche de ventes de propriétés approximativement équivalentes. Bien entendu, les experts des vendeurs ne manquaient pas

(32) AD Yvelines, 64 L 41, Gabriel Bain contre Adrien Mercier, 11 brumaire VII.

(33) Encore un mot.

(34) Les plaignants avaient cependant des alternatives, car la loi imposait que les cours prennent en considération les « facilités et avantages résultants des termes accordés pour le paiement » dans un contrat qui auraient pu favoriser l'une ou l'autre des parties. La plupart du temps il y avait un délai de plusieurs mois entre la signature du contrat et le paiement, offrant un « avantage » évident aux vendeurs. Les Conseils définirent finalement ces « facilités et avantages » le 24 prairial an VII. Jevardat-Fombelle, Conseil des Anciens, Rapport... concernant les rescisions, 24 prairial an VII; et J. CoRnudet, Conseil des Anciens, Opinion, 12 prairial an VII. 
de signaler la présence d'une belle cheminée comme preuve de la valeur élevée de la propriété et du préjudice évident causé par un prix déraisonnablement bas. Les experts des acquéreurs leur opposaient la pluie qui s'infiltrait par la toiture, et l'état négligé de la propriété au moment de la vente. Les experts devaient ensuite fixer le montant, toujours en assignats, des inévitables pots-de-vin et autres paiements illégaux en espèces.

Cette idée de baser l'estimation sur la vente de propriétés avoisinantes, et de la donner uniquement en assignat, heurta les experts et les vendeurs, qui la jugèrent totalement impossible ou tout simplement irréalisable. Dans plus d'une affaire, les experts bâclèrent complètement leurs calculs, les effectuant en espèces plutôt qu'en papier, ou en se référant aux tableaux de dépréciation plutôt qu'à des ventes locales, à tel point que toute la procédure dut être reprise. Si les deux experts n'étaient pas d'accord, un troisième était nommé pour régler le différend. Ensuite, le tribunal étudiait l'affaire de manière plus approfondie. Les juges estimaient si la date de conclusion des contrats de vente ou la date à laquelle les requêtes avaient été introduites entraient dans le cadre des limites fixées par les lois - treize affaires, au moins, soulevèrent des difficultés à cet égard. Ils examinaient la question de la recevabilité des ventes aux enchères. Finalement, les juges se prononçaient sur l'affaire, même si ces décisions ont laissé peu de traces dans les archives des juridictions civiles.

Malgré tous les obstacles mis en travers du chemin des plaignants, les documents existants révèlent qu'au moins 17 vendeurs quittèrent la juridiction civile en ayant remporté l'affaire, et qu'un autre gagna en appel. Six autres plaignants ont arraché davantage d'argent à leurs acquéreurs devant un notaire. Par conséquent, des 531 affaires traitées en Seineet-Oise, au moins 47 plaignants, ou quasiment $9 \%$, sortirent vainqueurs du bureau de paix, du tribunal civil ou à travers des accords rédigés devant un notaire. ${ }^{35}$ Par ailleurs, il ne serait pas surprenant de découvrir que d'autres cas ont été réglés à l'amiable, permettant ainsi aux deux parties d'échapper aux taxes et frais de dossiers.

\section{Le consentement contractuel en question}

« Le consentement des parties contractantes, qui est de l'essence du contrat de vente, consiste dans le concours de la volonté du vendeur de

(35) Je présume que de futures études plus approfondies mettront au jour des résultats plus éloquents. 
vendre telle chose à l'acheteur pour un tel prix, et de celle de l'acheteur d'acheter de lui ladite chose pour ledit prix ».

$(\text { Pothier) })^{36}$

La question ici va au-delà du simple fait de déterminer qui, des acquéreurs ou des vendeurs, méritaient le plus la compassion, et si leurs requêtes étaient véritablement fondées. Le débat porte en fait sur la notion du « consentement » telle que l'entendait le droit en vigueur à la fin du XVIII ${ }^{\mathrm{e}}$ siècle : les vives querelles au sujet de la lésion d'outre-moitié, qui divisaient à la fois la représentation nationale et les pamphlétaires mettaient en cause les notions mêmes de volonté et de consentement. Par conséquent, ces procès interrogeaient les concepts fondamentaux sur lesquels la loi contractuelle et la propriété moderne sont fondées. L'idée du contrat social, que se soit celle de Grotius, de Locke ou de Rousseau, était bien sûr à la base des conceptions révolutionnaires de la relation entre l'individu et l'État. La notion même de consentement était le point nodal de la loi révolutionnaire, et ce qui la différenciait des lois « barbares » de l'Ancien Régime. Davantage relevées cependant furent les nombreuses utilisations que firent les législateurs révolutionnaires de cette notion en tant que paradigme structurant des lois civiles. Selon un député, les séparations avaient forcé les femmes à renoncer à toute aide financière venant de leurs époux en échange de leur sécurité. Présentant les accords de séparation de l'Ancien Régime comme dénués d'un consentement contractuel libre, ou imposés sous la contrainte, un législateur s'exclama : «Et certes, un règlement d'intérêts, fait dans de pareilles circonstances, est trop contraire à la liberté qui doit régner dans toutes les conventions, pour devoir être maintenu ! ${ }^{37}$. Une nation libre, déclarèrent les députés, ne devait pas seulement tirer sa légitimité du caractère sacré du contrat social mais aussi de celle de ses contrats privés.

Toutefois, la notion de consentement contractuel autonome n'émergea pas de novo. Un des problèmes auxquels les législateurs révolutionnaires eurent à faire face était la quantité de concepts relatifs aux contrats qui s'étaient accumulés tout au long des siècles, associant plusieurs systèmes distincts et potentiellement incompatibles. Dans la loi romaine classique, les accords étaient essentiellement définis sur une base procédurale : les bons documents, rédigés correctement, enregistrés de

(36) Robert Joseph Pothier, Droit civil, op. cit., t. 1, p. 476.

(37) Pierre-Louis DUPRAT, Rapport sur la pétition de la citoyenne Rigoley, 30 pluviôse V. 
façon appropriée, et s'inscrivant dans les catégories adéquates ${ }^{38}$. Selon cette loi, le fait qu'un prix peu élevé soit spécifié dans un accord pouvait indiquer un cas de lésion ou peut-être un accord signé sous la contrainte ; et il n'y avait nul besoin d'enquêter pour déterminer comment les différentes parties interprétaient leur arrangement ${ }^{39}$. En France, l'équité - un concept tiré de la " loi naturelle idéalisée »- accepté à la fois par les canonistes de la fin de la période médiévale et par les civilistes du XVI ${ }^{\mathrm{e}}$ siècle, qui s'étaient réjoui de la dimension morale que ce concept apportait au droit - devint un élément important lors du traitement de ce genre affaires $^{40}$. D'après cette élaboration doctrinale, les contrats basés sur un intérêt réciproque nécessitaient un échange équilibré - l'équité. Par conséquent, la question essentielle permettant d'évaluer une convention était de savoir si le prix de l'objet échangé était véritablement « juste ». À la fin du XVII siècle, un troisième élément apparut de plus en plus fréquemment : la volonté des parties devenait le facteur le plus important dans l'interprétation d'un contrat ${ }^{41}$.

Il est possible que le grand juriste Jean Domat (1625-1696), qui voulait harmoniser les lois romaine, biblique, naturelle et coutumière, ait été le premier à défendre l'idée que dans la loi française, le consentement, et non le respect des procédures formelles, devait être le fondement de tout contrat ${ }^{42}$. Selon lui, les différentes formes d'accords volontaires «se forment mutuellement entre deux ou plusieurs personnes par leur volonté ;

(38) György DiósDI, Contract in Roman Law: From the Twelves Tables to the Glassators (1981); Alan WATSON, « The evolution of law : the Roman system of contracts, » Law and History Review, 2, 1984, p. 1-20.

(39) Shael Herman, «The uses and abuses of Roman law texts », The American Journal of Comparative Law, 29, autumn, 1981, p. 671-690.

(40) En anglais, la phrase « idealized natural law » vient de James GordLEY, qui argumenta que le concept d'équité était issu de concepts développés par Aristote qui furent tout d'abord remaniés par Thomas d'Aquin au XIII siècle, puis développés par les scolastiques au XVI ${ }^{\mathrm{e}}$ siècle : Foundations of Private Property Law, op. cit.; Odd Langholm, The Legacy of Scholasticism in Economic Thought: Antecedents of Choice and Power, Cambridge, Cambridge University Press, 1998. Sur la « moral theory of contract law », voir Howard J. BERMAN, « The religious sources of general contract law : an historical perspective », Journal of Law and Religion, 4, 1986, p. 103-124.

(41) Ce sujet mérite que l'on s'y attarde davantage. Voir Paul Viollet, Histoire du droit civil français, $2^{\mathrm{e}}$ éd., Paris, L. Larose \& Forcel, 1893, p. 590-608; Patrick Selim AтіуAн, The Rise and Fall of Freedom of Contract, Oxford, Clarendon Press, 1979; Shael Herman, « Uses and abuses », art. cit. ; Peter Birks, Grant McLeod, « The implied contract theory of quasi-contract : civilian opinion current in the century before Blackstone ", Oxford Journal of Legal Studies, 6, 1986, pp. 46-85; Samuel C. DAmren, "A 'Meeting of the minds': the greater illusion », Law and Philosophy, 15 (1996), 271-291.

(42) Jean Domat s'est probablement inspiré du passage dans le Digest, p. 50, 17, 23, legem enin contractus dedit. Shael Herman, «Uses and abuses », art. cit. 
ce qui se fait par les conventions, lorsque les hommes s'engagent mutuellement et volontairement dans les ventes $[\ldots] »^{43}$.

Pothier alla plus loin en suggérant que dans la loi romaine, le fait de considérer les seules procédures allait à l'encontre de la nature et de l'équité, insistant sur le fait qu'un contrat nécessitait une " chose », un prix, et plus particulièrement un consentement, " un concours des volontés $»^{44}$. De façon significative, l'apport majeur de Pothier concernait des domaines où le choix individuel jouait un rôle primordial - contrats de vente, obligations et successions ${ }^{45}$. Son style sec, ses références latines et ses démonstrations détaillées pourraient fatiguer un lecteur moderne. Mais on ne saurait sous-estimer combien ses travaux permettaient aux individus d'avoir une vision plus claire et bien plus concrète des modalités selon lesquelles ils pouvaient réaliser leurs propres objectifs en matière de propriété. Au cœur de son énumération aride des éléments nécessaires à un accord valable se trouvait l'affirmation croissante de l'individu et de ses désirs, caractéristique du siècle des Lumières.

$\mathrm{Au}$ début de la Révolution, les « économistes » minimisèrent l'importance de l'équité au sein des conventions, pour se focaliser sur le consentement, ce « concours des volontés ». Plus tard, devant la multiplication des appels en faveur du rétablissement des procès pour lésion, nombre de députés résistèrent, défendant la liberté contractuelle. Dans son projet de Code civil de 1796, Cambacérès proposa d'abolir tous les cas de lésion, au motif que ces procès ne prenaient pas en compte la claire acceptation, par les différentes parties, des conditions stipulées dans le contrat: « Nous avons dit que la convention n'existait pas sans un concours de volontés ; elle exige le consentement libre des parties ». Alors que les conceptions pré-révolutionnaires du droit des contrats permettaient l'exercice d'action pour lésion, il demanda aux législateurs d'y mettre un terme. La ruine économique - un sujet pénible durant l'automne 1796 était selon lui liée à ces pratiques antérieures :

(43) Jean Domat, Traité des lois, nouvelle édition, revue, corrigée et précédée d'une notice historique sur Domat par Joseph Remy, Paris, Firmin-Didot, C. Béchet, Roret..., 1828 ; réédition, Caen, Centre de philosophie politique et juridique, 1989 1989, p. 70.

(44) Robert Joseph Pothier, Droit civil, op. cit., t. 1, p. 4-5, 476. Voir la discussion de Pothier sur la nature de la possession, qui nécessitait non seulement que l'on possède l'objet mais également la "volonté de posséder » (animus possidendi) : Coutumes des duché, bailliage et prévôté d'Orléans, Nouvelle édition, Paris, Debure ; Orléans, Vve Rouzeau-Montaut, 1780, p. 832.

(45) Léopold ThÉZARD, De l'influence des travaux de Pothier et du chancelier d'Aguesseau sur le droit civil moderne, Paris, A. Durand, 1866. 
« La lésion avait le double inconvénient d'être une source intarissable de procès et de nuire aux progrès de l'agriculture ainsi qu'à l'embellissement des cités, par le caractère d'incertitude qu'elle donnait aux engagements. Notre législation doit au contraire imprimer le sceau de la stabilité aux actes faits sous ses auspices [...]».

Il affirma qu'un contrat signé en toute liberté ne devrait plus être considéré comme étant défectueux : «On se persuade difficilement qu’un propriétaire se trompe de moitié sur la valeur d'un héritage qu'il aliène $»^{46}$. Un chœur de législateurs appuya ses arguments en faveur du consentement contractuel, considérant qu'on pouvait laisser l'État intervenir pour protéger les victimes de fraude ou de coercition, mais non pour venir en aide à un vendeur imprudent. Le consentement, s'il avait été donné librement, constituait le seul et unique fondement du contrat.

De nombreux législateurs, pamphlétaires et juristes contestèrent ce point de vue. À lui seul, le consentement, ou du moins, le consentement dans son acception la plus simple, ne suffirait pas. Les tenants de l'interventionnisme pouvaient clamer, tout comme leurs opposants, l'adage de Pothier «res, pretium, consensus », mais ils savaient bien que celui-ci offrait lui-même une définition plus complexe de la notion de convention. N'avait-il pas insisté sur le fait que le prix devait être un prix sérieux et que « chacune des parties entend recevoir l'équivalent de ce qu'elle donne $»^{47}$ ? Par ailleurs, comme le firent remarquer les jurisconsultes les plus érudits, il était clairement spécifié dans les textes latins : " Si la moitié du vrai prix - verum pretium - n'a pas été payé ». Étant donné que les prix stipulés dans les contrats étaient en assignats, et que ce dernier perdait rapidement de la valeur, ils ne pouvaient donc pas représenter un 《 vrai prix payé ${ }^{48}$. Si les propriétaires avaient pu prévoir de vendre leur propriété pour un certain montant, ils n'auraient pas pu se douter que l'assignat allait chuter si vite. Et étant donné qu'il y avait un délai de quatre mois en moyenne entre la signature du contrat et le premier versement, alors que pendant ce temps, la valeur du papier-monnaie continuait à dégringoler, le vendeur se trouvait d'autant plus désavantagé. Sans équité, il ne pouvait y avoir de consentement.

(46) « Suite du Discours préliminaire », Moniteur, 13 fructidor an IV.

(47) Cité dans: Olivier, Homme de Loi, Rentes perpétuelles et viagères (constituées en assignats) ou Réfutation des On Dit et des erreurs... Paris, chez l'auteur, s. d.

(48) Encore un mot, op. cit. 
Les résolutions votées durant l'an VI et l'an VII, ont ainsi établi un ensemble de principes régissant l'intervention de l'État dans un large éventail d'accords contractuels. Elles mirent en avant l'idée que ce dernier n'entraverait nullement la liberté contractuelle en modifiant les contrats, ou en rétablissant l'équité, mais ne ferait au contraire que restaurer l'intention initiale des parties contractantes : « Ce n'est pas porter atteinte aux conventions, c'est en assurer l'exécution, c'est donc les protéger, affirma un rapporteur, ainsi le droit de porter la loi proposée existe dans ce législateur ; l'équité exige qu'il en use $»^{49}$. «La vicissitude des faits a changé la condition des parties », expliqua Claude-Ambroise Regnier, « et elle exige qu'on les place dans l'état où elle eussent été, si elles eussent contracté en numéraire $\gg^{50}$. Il était du ressort des représentants de fournir réparation pour ces contrats, lorsque les paiements stipulés étaient insuffisants. Cette vision était en accord avec les principes établis par la constitution de l'an III, à savoir que les bases d'un gouvernement sain ne résidaient pas dans la nature mais dans la société civile et la $\operatorname{loi}^{51}$. La propriété - ses limites, définitions et utilisations - ne procédait que du législateur. Loin d'un acte d'une constitutionalité douteuse, les Conseils rétablissaient la justice même.

Même dans le Code civil de 1804, la lésion était toujours considérée comme un motif valable lors des actions en dommages et intérêts ${ }^{52}$. Ces justifications exigeaient cependant une analyse encore plus minutieuse des composantes du consentement. Thomas-Laurent Mouricault retraça l'historique des différentes approches des codes de Domat jusqu'à Pothier, soulignant le fait que le consentement donné librement était la base du contrat : « Le consentement des parties contractantes est mis à la tête, et avec raison, puisque toute convention suppose la réunion des volontés de ceux qui y interviennent $\rangle^{53}$. Faisant écho à Domat et Pothier, il ajoutait : « La volonté doit être le produit d'une détermination libre autant que réfléchie $[. .$.$] . Le consentement qui n'a été donné que par l'effet de l'er-$ reur, du dol ou de la violence n'est donc pas un consentement réel et an IV.

(49) [CRetet], Rapporteur, Conseil des Anciens, AN, C 489, fol. 64, n 239, 9 messidor

(50) AN, C 520, fol. 77, $\mathrm{n}^{\circ}$ 55, Conseil des Anciens, 2 frimaire an VI.

(51) Françoise Fortunet, « Des droits et des devoirs », dans La Constitution de l'an III, ou, L'ordre républicain : actes du colloque de Dijon, 3 et 4 octobre 1996, Jean Bart et alii (dir.), Dijon : Editions universitaires de Dijon, 1998, p. 17-28.

(52) Articles 1674-1685.

(53) Thomas-Laurent Mouricault, Discours prononcé... sur le titre... Des contrats et des obligations conventionnelles en général, 17 pluviôse an XII. 
valable ». Cependant les formes de violence, de fraude ou d'erreur ne suffisaient pas toutes à infirmer une convention. L'erreur devait concerner « la substance même de la chose, ou [...] le motif déterminant de l'engagement ». Par ailleurs, si un consentement était donné par peur de représailles, la menace devait être de nature à intimider " une personne raisonnable $»^{54}$.

Guillaume-Jean Favard de Langlade s'appliqua également à expliquer les conséquences de ces erreurs sur un contrat néanmoins valide. D'une façon ou d'une autre, un individu, même lorsqu'il se trompait, devait être considéré comme capable de raisonner, et par conséquent, de donner son consentement. Sinon, le contrat lui-même serait annulé, plutôt que simplement résilié. En cas de résiliation, un contrat demeurait essentiellement valide, mais il était nécessaire que l'on établisse un nouveau prix. Jouant habilement sur les mots, il expliqua qu'en cas d'erreur, « il faut que l'erreur tombe sur la substance même de la chose qui est l'objet de la convention : si elle tombait sur la personne, elle n'annulerait la convention qu'autant que la considération de la personne en aurait été la cause principale $\aleph^{55}$. Quant au responsable de l'erreur, ce n'était pas la personne qui avait subi le tort. Au lieu de cela, alors même qu'il n'y avait pas eu de fraude, l'erreur élevée au rang de lésion était néanmoins « bien ordinairement le fruit de manœuvres adroites approchant du dol». Par conséquent, laissant la capacité de raisonnement des victimes essentiellement intacte, le code était en mesure d'établir qu'un contrat comportant de telles erreurs pouvait faire l'objet d'actions pour lésion ${ }^{56}$.

Il semble bien que ce raisonnement l'ait emporté. Charles Toullier, doyen de la faculté de droit de l'Université de Rennes, expliqua qu'un contrat dans lequel il y avait lésion était malgré tout fondé sur le consentement. Selon lui, la « lésion ne rend pas les conventions nulles dans leur principe parce qu'elle ne détruit pas le consentement, mais elle donne lieu quelquefois à la résolution du contrat $»^{57}$.

Ces procès marquent assurément une étape dans l'histoire du droit français des contrats. Ils pourraient répondre aux attentes d'un historien cherchant à mettre en lumière l'émergence de la propriété privée moderne,

(54) Mouricault, Des contrats.

(55) Guillaume-Jean FAVARD de LANGLADE, Rapport [...] sur les trois premiers chapitres du titre du $3^{\mathrm{e}}$ livre du Code civil, Tribunat, Séance du 13 pluviôse XII.

(56) Ibidem.

(57) Droit civil français, 6, p. 106. 
dépourvue de ses attributs de l'Ancien Régime. Au plan législatif, il importait peu que la propriété ait eu à l'origine un statut d'acquêts ou successorale; la loi s'attachait uniquement à la valeur marchande du bien. De plus, au grand étonnement de nombreux juges civils, la loi semblait rassembler plusieurs formes de transactions précédemment distinctes dans une seule et même catégorie, celle d' "immeubles ». Fournissant davantage de soutien à ceux qui se plaçaient du côté du capitalisme naissant, les législateurs, quelle que soit leur position dans le débat, s'accordèrent à rejeter toutes les estimations de la propriété dérivée des contrats plus stables de l'Ancien Régime. À rebours de celles-ci, la propriété était ici généralement considérée comme soumise aux fluctuations du marché, même si celles-ci étaient extrêmes. Pour certains députés, la valeur d'une propriété devait être fondée sur son rendement locatif ou sur le revenu agricole $^{58}$. En insistant, dans la version finale de la loi, sur le fait que les experts ne devaient utiliser que des prix en assignats, et se fonder sur les ventes locales de la même époque, ils faisaient du marché la seule source de la valeur.

Les cas pratiques nous offrent un tableau plus mitigé. Pour les vendeurs, contrairement aux législateurs, la provenance du bien était toujours très importante. Le désignant comme patrimoine ou héritage, les anciens propriétaires, ainsi que leurs héritiers, étaient fermement résolus à récupérer leur bien, même si celui-ci était modeste. Par conséquent, ces cas donnent une vision plus nuancée de la conception moderne des droits de propriété, ceux-ci évoluant au gré des balbutiements du marché et de la culture de marchés9.

Toute tentative de distinguer les vendeurs et les acheteurs sur la base de leur statut social est vouée à l'échec. Quelques résidences imposantes, et quelques rentiers miraculeusement chanceux, apparaissaient à côté de spéculateurs infortunés. Mais généralement, les plaignants comme les défen-

(58) Ceci aurait été conforme à de nombreuses pratiques datant de l'Ancien Régime: Encore un mot, op. cit.; Livessan, Ultimatum sur l'exercice de l'action en rescision pour cause de lésion d'outre moitié... Paris, Imprimerie des Annales d'agriculture, (s. d.) ; Observations sur le mode et les effets de l'action afin de restitution pour cause de lésion d'outre moitié, Paris, Imprimerie de Belin, s. d.

(59) Jean-Pierre Hirsch, Les deux rêves du Commerce: Entreprise et institution dans la région lilloise (1780-1860), Paris, Éditions de l'EHESS, 1991 ; William M. REDDY, The Rise of Market Culture : The Textile Trade and French Society, 1750-1900, Paris-Cambridge, Éditions de la MSH-Cambridge University Press, 1984; Philippe Minard, La Fortune du colbertisme: état et industrie dans la France des Lumières, Paris, Fayard, 1998 ; Judith A. Miller, Mastering the Market: The State and the Grain Trade in Northern France, 1700-1860, Cambridge, Cambridge University Press, 1998. 
deurs, étaient des commerçants, des veufs, ou de plus modestes cultivateurs, vignerons et journaliers. Leur propriété consistait le plus souvent en quelques arpents de terre, ou une " petite maison et dépendances », situés à un emplacement très quelconque. Même si elles existaient, les propriétés constituées de 50 ou 100 perches de terre, de vignes ou de bois taillés étaient moins fréquentes. Les nombreuses demeures versaillaises bordant le boulevard de l'Égalité et la rue J.-J. Rousseau qui apparaissent dans ces procès nous donnent un aperçu de la vitesse à laquelle l'économie de la ville s'affaiblit après 1791, et de la rapidité avec laquelle l'annonce du vote de nouvelles lois circula parmi les anciens résidents, une information probablement relayée par ces entreprenants hommes de loi, systématiquement présents au côté des plaignants dans les tribunaux.

Il serait cependant difficile de déterminer qui, des vendeurs ou des acheteurs, étaient les plus prospères avant la Révolution. Ceux qui avaient été forcés de vendre leurs demeures, le plus souvent parce que leurs créanciers commençaient à leur tourner autour, étaient-ils plus favorisés ou plus vulnérables avant l'hyperinflation ? On peut percevoir la ténacité des nombreux groupes d'héritiers qui remplirent les tribunaux, déterminés à réclamer les parcelles de terre que leurs parents, vieillissants, avaient progressivement vendu, alors que les circonstances s'aggravaient. Cependant, le fait qu'ils aient tout de même possédé une propriété à vendre les plaçait parmi les franges les plus favorisées de la société française. Peutêtre que les acheteurs se trouvaient continûment les plus avantagés. Ont-ils tourné à leur avantage leur situation financière légèrement plus favorable, accaparant quelques lopins de terre, consolidant leurs avoirs, ou acquérant la résidence plus grande dont ils avaient toujours rêvé ? Ou bien une fortune changeante leur avait-elle permis de profiter de quelque avantage inattendu ? Les archives ne sont pas d'un grand secours pour trancher, et suggèrent plutôt que la propriété était transmise à l'intérieur de groupes sociaux plus ou moins similaires, et le plus souvent sur la base de réseaux commerciaux ou de relations de voisinage. Il est également possible, à en juger par les professions répertoriées, que les vendeurs aient été à un moment donné légèrement plus prospères que leurs acquéreurs, mais pas nécessairement. Les ventes se faisaient des garde-moulins à des marchands de bois, des veuves de marchands de vin retraités à des tonneliers, des vignerons à des cultivateurs, et des hommes de loi à des pâtissiers et des perruquiers, ou d'anciens aubergistes à des serruriers.

Malgré toutes les questions qui restent en suspens, ces conflits législatifs acerbes au sujet de toutes sortes de transactions entre particuliers, tout comme les procès pour lésion, témoignent du choc provoqué par 
l'hyperinflation révolutionnaire. À chaque chute de l'assignat, la pression montait dans les foyers : où se procurer la nourriture pour le mois à venir? Comment faire pour payer les créanciers ? La vente de propriétés, et celle des biens nationaux, a assurément été profondément déstabilisante, puisqu'elle a remis en question tous les calculs, même les plus minutieux. Elle a ébranlé le sentiment de sécurité qu'éprouvaient les familles aisées et a broyé les plus pauvres. Les délais de promulgation des lois - compréhensibles et inévitables - signifiaient en outre que le doute allait planer au-dessus de chaque type d'accord pour plusieurs années consécutives.

Chaque nouvelle résolution pouvait faire renaître les espoirs, ou au contraire les anéantir. Lorsqu'une résolution finissait par être votée, les tribunaux étaient assaillis par les parties venant régler, ou contester, chaque type de comptes, livres de comptes ou de contrats. L'enthousiasme avec lequel les plaignants de Seine-et-Oise accueillirent les procès pour lésion, ainsi que le nombre et le taux relativement élevé des succès, indiquent à quel point l'hyperinflation avait semé le doute parmi les propriétaires de biens. Non seulement les maisons, les fermes et les bois avaient été vendus rapidement, mais pendant au moins une dizaine d'années, tandis que les affaires se succédaient devant les tribunaux, la validité de ces ventes a été remise en question. Pour un cas tranché par les tribunaux, beaucoup d'autres étaient certainement réglés à l'amiable. Un bon nombre d'autres acquéreurs devaient probablement craindre que leurs vendeurs ne les attaquent en justice, tandis que ces derniers nourrissaient l'espoir de récupérer leur héritage perdu. À côté des quelque cinq cent plaignants ici repérés, il y en eut probablement des milliers d'autres dans le département, n'osant pas se porter en justice, qui regardaient sombrement leur ancienne demeure, mesurant l'ampleur de leur perte, et à quel point leur vie avait été bouleversée. Et combien d'autres à travers toute la France ? Le coût total de l'hyperinflation a été à peine reconnu ; toutefois on commence à entrevoir son bilan.

Judith A. MiLLER

Département d'histoire

Bowden Hall 219

Emory University

Atlanta, GA 30322

États-Unis

histjam@emory.edu
Traduit de l'anglais (États-Unis) par Sophie Bond, avec le concours de Philippe Minard sophiebond@free.fr 